
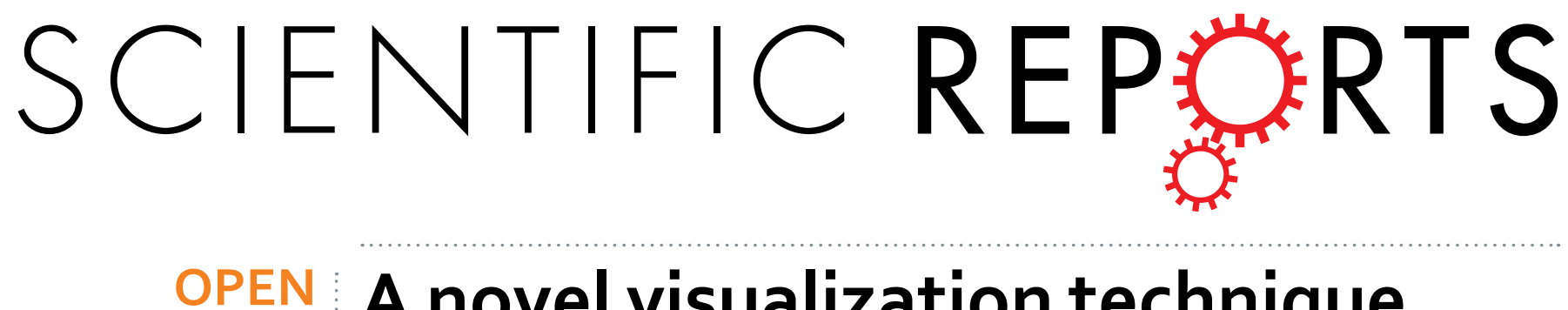

\title{
A novel visualization technique for measuring liquid diffusion coefficient based on asymmetric liquid-core cylindrical lens
}

Received: 08 March 2016

Accepted: 02 June 2016

Published: 21 June 2016
Licun Sun \& Xiaoyun Pu

A visualization and quantification optical method for measuring binary liquid diffusion coefficient $(D)$ based on an asymmetric liquid-core cylindrical lens (ALCL) is introduced in this paper. Four groups of control experiments were performed to verify the influences of diffusing substance category, concentration and temperature on diffusion process, and the measured $D$ values were well consistent with data measured by Holographic interferometry and Taylor dispersion methods. The drifting of the diffusion image recorded by CCD reflects the diffusion rate visually in an easily understandable way. This optical method for measuring $D$ values based on the ALCL is characterized by visual measurement, simplified device, and easy operation, which provides a new way for measuring liquid $D$ value visually.

Diffusion is a process which leads to an equalization of concentrations within a single phase as a result of random molecular motions ${ }^{1}$. The study of diffusion is important in chemical engineering and other fields, such as biological systems, pollution control and separation of isotopes ${ }^{2}$. Diffusion coefficient $(D)$ measurements provide fundamental information needed in various engineering and industrial operations, including the design of chemical reactors, liquid-liquid extractors, absorbers, distillation columns and so $\mathrm{on}^{3}$. In addition, the knowledge about diffusivity is also needed for the study of fluid-state theory, mass-transfer phenomena, and molecular interactions ${ }^{4}$.

Diffusion phenomena can appear in all gaseous, liquid and solid materials ${ }^{1,5}$. The color of a wall turns darker if a pile of coal is beside it for a long time in the absence of the external forces; the clear water becomes colored gradually when the iodine solution contacts with it without stirring; the scent of rose can be smelled even though there is no wind. Those are all diffusing phenomena, intuitive and easy to feel. Nevertheless, if two kinds of colorless transparent liquids are placed in contact, how can we present the diffusion process vividly and measure the $D$ value accurately?

There are many well-established methods for the determination of liquid $D$ value. Holographic interferometry $^{6}$, diaphragm cell ${ }^{7}$ and Taylor dispersion ${ }^{8}$ are the most widely used techniques for diffusivity studies among them. Holographic interferometry method needs to analyze the interference fringes to work out the concentration profile of diffusive solutions ${ }^{9}$; diaphragm cell method requires to measure the initial and final concentrations of the upper and lower cell compartments ${ }^{10}$; Taylor dispersion method uses a refractive index detector to obtain a concentration profile that is similar with the Gaussian distribution curve ${ }^{11}$. Those three methods neither provide direct observation of the dynamic diffusion processes nor can be understood easily. Besides the common disadvantages mentioned above, interferometry method is time-consuming and requires extremely strict experimental environment ${ }^{12}$; the disadvantages of the diaphragm cell are also time-consuming and the necessity for calibration of the cell ${ }^{13}$; Taylor dispersion method makes use of a spiral capillary with length up to several meters and the mobile phase is required to flow through the round cross section at a constant flowing rate, which is difficult to achieve and leads to poor accuracy ${ }^{14}$.

To overcome these disadvantages, an asymmetric liquid-core cylindrical lens (ALCL), used as both diffusion pool and key imaging element, has been designed and used to measure liquid $D$ values by our group recently ${ }^{15,16}$. ALCL can be used to measure refractive index (RI) of filled liquids in the way of spatial resolution along the axis of ALCL. If a dynamic gradient distribution of RI forms along the axis of ALCL because of molecule diffusion, ALCL projects a dynamic "beam waist" image on the CCD. The dynamic image reflects the diffusion process

Department of Physics, Yunnan University, Kunming, Yunnan 650091, China. Correspondence and requests for materials should be addressed toX.P. (email: xypu@163.com) 


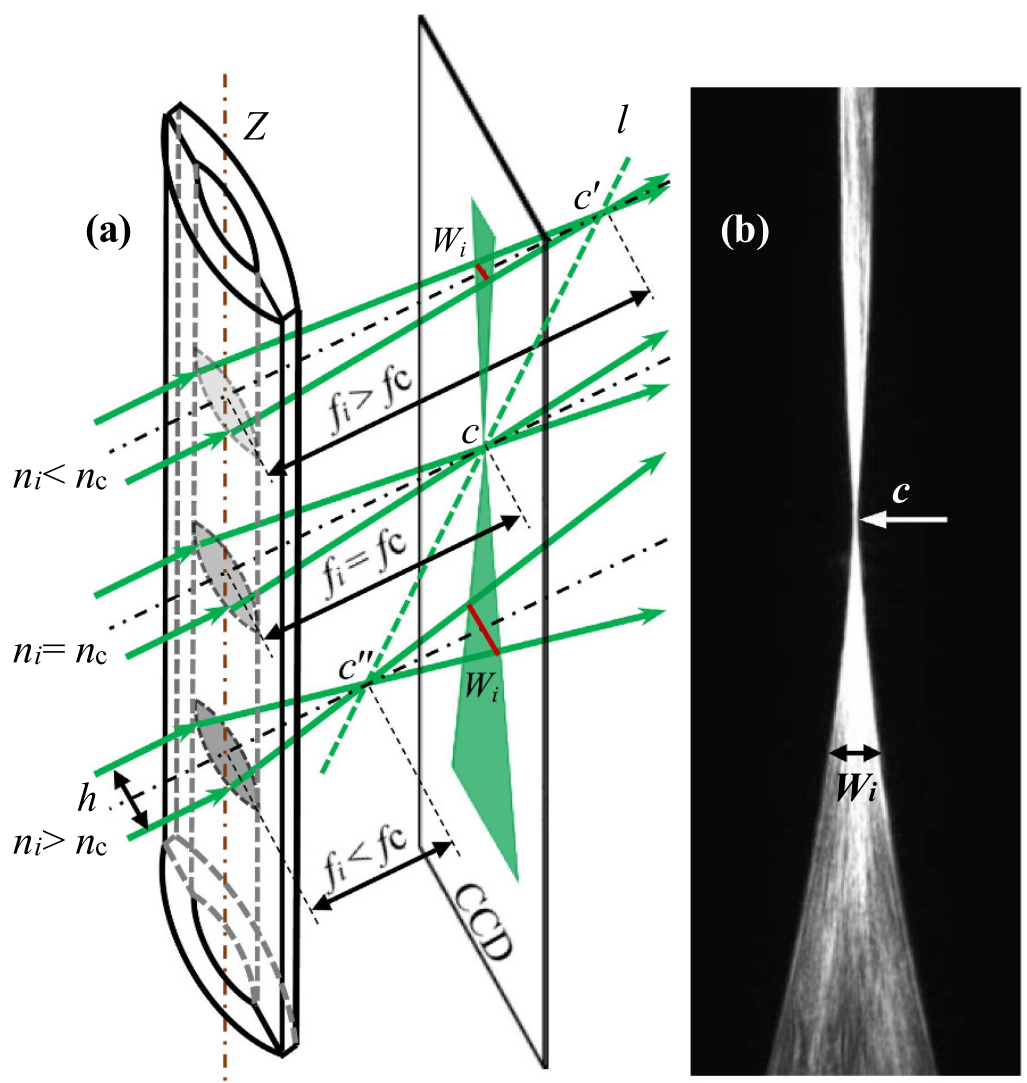

Figure 1. Imaging principle of ALCL. (a) a sloping focal curve $(l)$ forms behind ALCL when a RI gradient distribution of the filled liquid is formed along $Z$-axis. (b) an experimental image recorded by CCD. The point $c$ is the only clear imaging point relative to the thin liquid layer of $n_{i}=n_{c}$, and $W_{i}$ is the projected width when $n_{i}$ is not equal to $n_{\mathrm{c}}$.

vividly, and the $D$ value, based on the Fick's second law, can be figured out either by recording the locations of "waist" at different times ${ }^{15}$ or by analyzing a simultaneous diffusion image rapidly ${ }^{16}$.

In this paper, we demonstrated the validity of our method to measure liquid $D$ values through theoretical discussion and experimental results. The ALCL was utilized in the diffusion experiments of ethylene glycol-water (EG-water) and triethylene glycol-water (TEG-water) systems, the influences of solution concentration and temperature on diffusion were investigated, related $D$ values were measured. Equipped with the ALCL and a CCD camera, all of diffusion processes can be visualized and displayed in the attached visualization files.

\section{Methods}

Imaging principle for ALCL filled with diffusion solution. The imaging principle of ALCL filled with diffusion liquids is showed in Fig. 1(a). The iterative relations between the focal length $f_{i}$ and RI of liquid $\left(n_{i}\right)$ filled in the ALCL can be written as,

$$
\begin{gathered}
f_{i}=\frac{R_{4} S_{4}}{n_{0} R_{4}+\left(n_{0}-1\right) S_{4}}+d_{3}+d_{2} / 2, \\
S_{4}=\frac{n_{0} R_{3} S_{3}}{n_{i} R_{3}+\left(n_{i}-n_{0}\right) S_{3}}-d_{3}, \\
S_{3}=\frac{n_{i} R_{2} S_{2}}{n_{0} R_{2}+\left(n_{i}-n_{0}\right) S_{2}}-d_{2} \\
S_{2}=\frac{n_{0} R_{1}}{n_{0}-1}-d_{1} .
\end{gathered}
$$

Here the curvature radii of the four refraction surfaces of ALCL are $R_{1}=20.0 \mathrm{~mm}, R_{2}=R_{3}=17.0 \mathrm{~mm}$, and $R_{4}=37.6 \mathrm{~mm}$, respectively. The wall thicknesses and cavity thicknesses are $d_{1}=d_{2}=d_{3}=3 \mathrm{~mm}$, respectively. The material of cylindrical lens is $\mathrm{K} 9$ glass $\left(n_{0}=1.5163\right.$ at $\left.\lambda=589 \mathrm{~nm}\right)$. If parameter $f_{i}$ is determined by measurement 


\begin{tabular}{|l|l|l|l|}
\hline Experiment (a) & Experiment (b) & Experiment (c) & Experiment (d) \\
\hline EG diffusing in pure water at $25^{\circ} \mathrm{C}$. & TEG diffusing in pure water at $25^{\circ} \mathrm{C}$. & EG diffusing in pure water at $35^{\circ} \mathrm{C}$. & EG diffusing in $50 \%$ aqueous EG at $25^{\circ} \mathrm{C}$. \\
\hline$C_{1}=1.0, C_{2}=0$ & $C_{1}=1.0, C_{2}=0$ & $C_{1}=1.0, C_{2}=0$ & $C_{1}=1.0, C_{2}=0.50$. \\
\hline
\end{tabular}

Table 1. Contents of four experiments.

in advance, RI of liquid $\left(n_{i}\right)$ can be computed based on Eqs $(1 \sim 4)$. The main purpose for designing the ALCL is to maintain a good RI measurement sensitivity and reduce spherical aberration to the minimum. The experimental setup and the method for measuring liquid RI based on the ALCL have been introduced in detail in refs 15 and 16.

Two kinds of diffusion solution are filled into the ALCL sequentially with the dense solution occupying the lower half. Once two solutions contact, the diffusion process commences. Dynamic gradient distributions of the concentration for the mixed solution are formed gradually along the diffusion direction defined as $Z$-axis. The focal length $\left(f_{\mathrm{i}}\right)$ of ALCL for different thin liquid layer changes with the layer's RI $\left(n_{i}\right)$. The collimation light will converge to be a sloping curve $(l)$ after it passing through the ALCL filled with the diffusion solutions. If a CCD is fixed at the focal plane of the ALCL filled with liquid of RI $=n_{c}$, as shown in Fig. 1(a), the curve $l$ will only intersect with the image plane (CCD) at the point $c$, while the collimated beams passing the ALCL filled with liquid of $\mathrm{RI}=n_{i}>n_{\mathrm{c}}\left(\right.$ or $n_{i}<n_{\mathrm{c}}$ ) shall project a width $W_{i}$ on the CCD plane. Figure $1(\mathrm{~b})$ is the experimental image recorded by the CCD, consistent with the theoretical analysis. Let $h$ be the width of collimated light beams, $f_{\mathrm{c}}$ and $f_{i}$ be the focal length of the ALCL when filled with liquid of $\mathrm{RI}=n_{\mathrm{c}}$ and $\mathrm{RI}=n_{i}$, respectively, from the view of geometrical optics, the image width $\left(W_{i}\right)$ and the focal length $\left(f_{i}\right)$ satisfy

$$
\frac{h / 2}{f_{i}}=\frac{W_{i} / 2}{\left|f_{i}-f_{\mathrm{c}}\right|}
$$

On the condition that $h$ and $f_{\mathrm{c}}$ being known, $f_{i}$ can be calculated by Eq. (5) when $W_{i}$ is read out straightforwardly by a software built in the CCD camera. Inserting $f_{i}$ to Eqs $(1 \sim 4), n_{i}$, the RI of liquid filled in ALCL can be calculated. Since $W_{i}$ value is varied with the $Z$-axis as shown in Fig. $1, W_{i}=W_{i}(Z)$, after $f_{i}=f_{\mathrm{i}}(Z)$ being determined by experimental method, $n_{i}=n_{i}(Z)$ and $C_{i}=C_{i}(Z)$, i.e., the spatial distribution of RI and concentration can be determined completely by only one diffusion image.

Theoretical and computational methods for diffusion coefficients. Assuming binary solutions involved in diffusion to be $\mathrm{A}$ and $\mathrm{B}$, diffusion direction to be $Z$-axis and $C(Z, t)$ to be the mass fraction of $\mathrm{A}$ in $\mathrm{B}$ at diffusion time $t$ and position $Z$, based on Fick's second law, $C(Z, t)$ satisfies the following equation ${ }^{3}$ :

$$
\frac{\partial C(Z, t)}{\partial t}=D \frac{\partial^{2} C(Z, t)}{\partial Z^{2}}
$$

where, $D$ is the diffusion coefficient. Assuming the initial concentrations to be $C_{1}$ and $C_{2}$ on each side of the contact interface $(Z=0)$ before the diffusion begins, according to the boundary and initial conditions, the solution of Eq. (6) is an error function that can be expressed as ${ }^{17}$

$$
C(Z, t)=\frac{C_{1}+C_{2}}{2}+\frac{C_{1}-C_{2}}{2} \operatorname{erf}\left(\frac{Z}{2 \sqrt{D t}}\right) .
$$

where, $\operatorname{erf}(u)=\frac{2}{\sqrt{\pi}} \int_{0}^{u} \exp \left(-t^{2}\right) d t$ is the Gauss error function ${ }^{18}$. Equation (7) can be rewritten by the inverse error function as

$$
Z=2 \sqrt{D} \operatorname{erfinv}\left\{\left[g[n(Z, t)]-\frac{C_{2}+C_{1}}{2}\right] /\left[\frac{C_{2}-C_{1}}{2}\right]\right\} \cdot \sqrt{t}-\Delta Z_{0} .
$$

Here, $g[n(Z, t)]=C(Z, t)$ relating solution concentration to its RI, can be pre-determined experimentally. $\Delta Z_{0}$ is a fixed deviation of the position $Z$ read out in actual measurements, as the contact interface cannot be an exact horizontal plane due to the intermolecular attraction. $C_{1}$ is set to be 1 , since pure diffusion chemical is filled in the lower part of ALCL, and $C_{2}$ is a destination concentration filled on the upper part of ALCL.

The location $\left(Z_{i}\right)$ of a selected thin liquid layer, which has a fixed concentration and then a fixed $\mathrm{RI}=\left(n_{\mathrm{c}}\right)$, varies with diffusion time $\left(t_{i}\right)$, and the $D$ values can be obtained by fitting linearly $Z_{i}$ and $\sqrt{t_{i}}$ values and comparing it with Eq. (8), since $\left[g[n(Z, t)]-\left(C_{1}+C_{2}\right) / 2\right] /\left[\left(C_{1}-C_{2}\right) / 2\right]$ is a constant when the thin liquid layer is selected. The drift velocity of "waist" image reflects the diffusion rate intuitively.

\section{Results}

To study the effect of diffusing substance category, concentration and temperature on diffusion process, four contrast experiments have been carried out, and the experimental contents are listed in Table 1.

The experiment (a) is set as a contrast, and only one variable is changed in the other experiments. A CCD camera is regulated in a suitable vertical location, making the focus points (the "waist" of each image) of the four experiments at the same height in the initial recording time, as shown in Fig. 2(a). Dynamic concentration gradient distribution will form in the core area of ALCL due to the diffusion process, moreover, the focus point will drift with time as showed in Figs 3 and 4. The drift speed of the "waist" demonstrates the diffusion rate. 


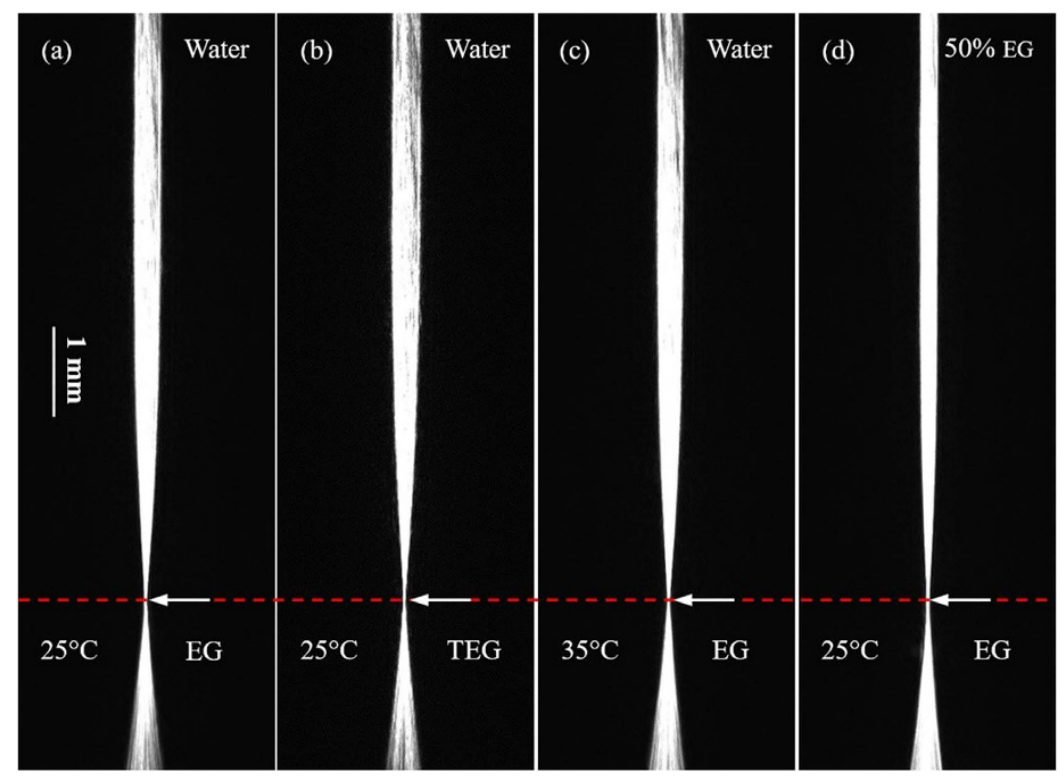

Figure 2. The images taken at the diffusion time of 10 minutes. (a) EG diffusing in pure water $\left(C_{1}=1\right.$, $\left.C_{2}=0\right)$ at $25^{\circ} \mathrm{C}$. (b) TEG diffusing in pure water $\left(C_{1}=1, C_{2}=0\right)$ at $25^{\circ} \mathrm{C}$. (c) EG diffusing in pure water $\left(C_{1}=1\right.$, $\left.C_{2}=0\right)$ at $35^{\circ} \mathrm{C}$. (d) EG diffusing in $50 \%$ aqueous EG $\left(C_{1}=1, C_{2}=0.5\right)$ at $25^{\circ} \mathrm{C}$. The arrows indicate the "waist" positions.

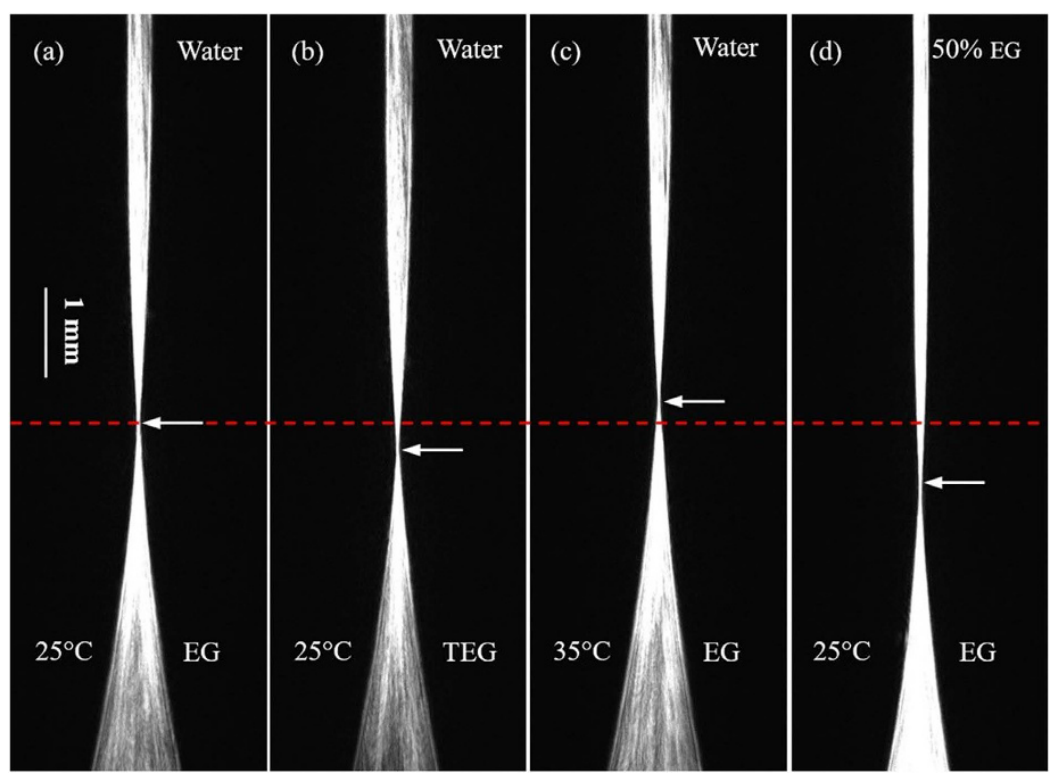

Figure 3. Same as Fig. 2, but the images were taken at the diffusion time of 45 minutes.

Comparing the experiment (b) with the experiment (a), it is clear that the larger molecule $\left(\mathrm{MW}_{\mathrm{TEG}}=\right.$ $150.18>\mathrm{MW}_{\mathrm{EG}}=62.07$ ) has the larger friction among diffusion molecules, as a result, the larger molecule (TEG) has a lower diffusion rate as shown in Figs 3 and 4. The comparison between the two diffusion processes is displayed in the Supplementary Video S1.

Comparing the experiment (c) with the experiment (a), it is clear that higher temperature causes more vigorous random molecular motions, leading to a higher diffusion rate as shown in Figs 3 and 4 . The comparison between two diffusion processes is displayed in the Supplementary Video S2.

Comparing the experiment (d) with the experiment (a), we can see that a dense diffusion solution gives rise to a short mean free path, leading to a low diffusion rate as shown in Figs 3 and 4 . The comparison between two diffusion processes is displayed in the Supplementary Video S3.

In order to calculate $D$ values, the "waist" locations varied with diffusion time in the four experiments are listed in Table 2, in which $N_{i}$ means the number of pixel between the interface and the "waist" position and $Z_{i}$ is equal to $N_{i}$ multiplied by the pixel size $(3.45 \mu \mathrm{m}$ in this experiment). The RIs and mass fractions of the thin liquid 


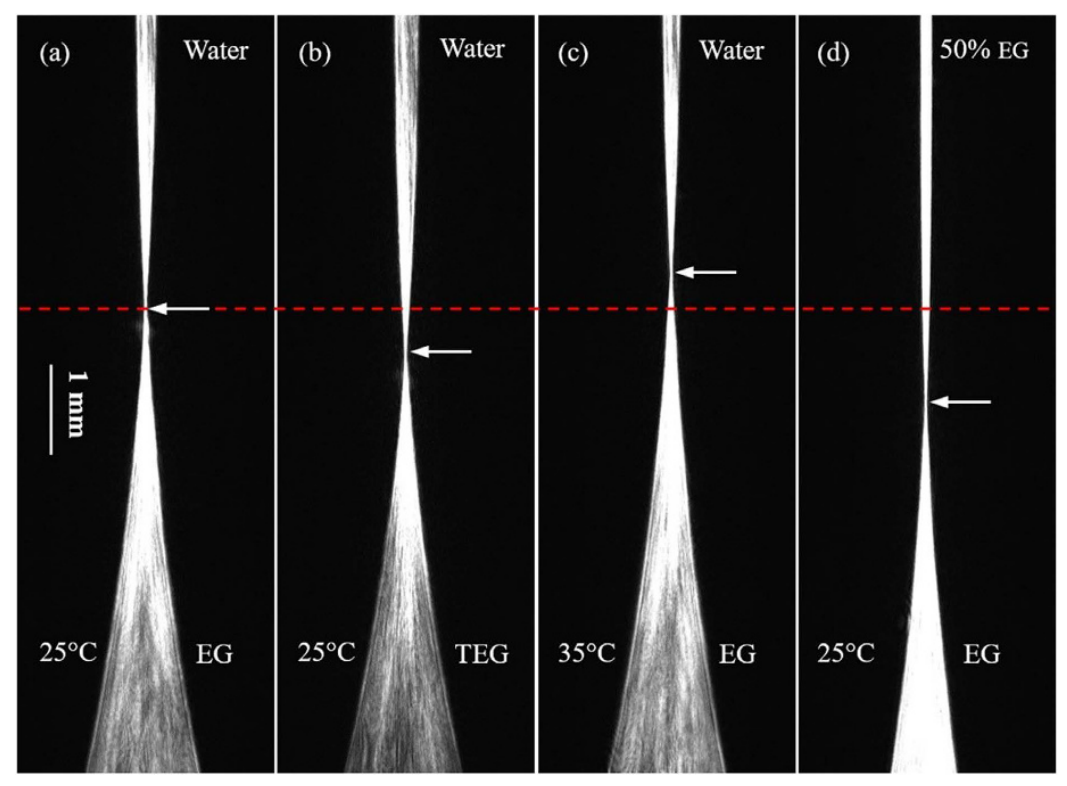

Figure 4. Same as Fig. 2, but the images were taken at the diffusion time of 90 minutes.

\begin{tabular}{|c|c|c|c|c|c|c|c|c|c|}
\hline \multirow{2}{*}{\multicolumn{2}{|c|}{ RI of liquid layer $\left(n_{c}\right)$}} & \multicolumn{2}{|c|}{ Experiment (a) } & \multicolumn{2}{|c|}{ Experiment (b) } & \multicolumn{2}{|c|}{ Experiment (c) } & \multicolumn{2}{|c|}{ Experiment (d) } \\
\hline & & \multicolumn{2}{|c|}{1.3391} & \multicolumn{2}{|c|}{1.3397} & \multicolumn{2}{|c|}{1.3379} & \multicolumn{2}{|c|}{1.3898} \\
\hline \multicolumn{2}{|c|}{ Mass fraction (\%) } & \multicolumn{2}{|c|}{5.72} & \multicolumn{2}{|c|}{5.66} & \multicolumn{2}{|c|}{5.67} & \multicolumn{2}{|c|}{55.65} \\
\hline \multicolumn{2}{|c|}{$C=g[n(z, t)]$} & \multicolumn{2}{|c|}{$9.8478 n-13.130$} & \multicolumn{2}{|c|}{$7.9082 n-10.538$} & \multicolumn{2}{|c|}{$10.0596 n-13.402$} & \multicolumn{2}{|c|}{$9.8478 n-13.130$} \\
\hline$t_{i} / \mathrm{s}$ & $\sqrt{t_{i}}$ & $N_{i}$ & $Z_{i} / \mu \mathrm{m}$ & $N_{i}$ & $Z_{i} / \mu \mathrm{m}$ & $N_{i}$ & $Z_{i} / \mu \mathrm{m}$ & $N_{i}$ & $Z_{i} / \mu \mathrm{m}$ \\
\hline 600 & 24.4949 & 548 & 1890.60 & 548 & 1890.60 & 548 & 1890.60 & 548 & 1890.60 \\
\hline 900 & 30.0000 & 652 & 2249.40 & 643 & 2218.35 & 705 & 2432.25 & 616 & 2125.20 \\
\hline 1200 & 34.6410 & 772 & 2663.40 & 744 & 2566.80 & 828 & 2856.60 & 694 & 2394.30 \\
\hline 1500 & 38.7298 & 873 & 3011.85 & 815 & 2811.75 & 946 & 3263.70 & 747 & 2577.15 \\
\hline 1800 & 42.4264 & 949 & 3274.05 & 883 & 3046.35 & 1035 & 3570.75 & 796 & 2746.20 \\
\hline 2100 & 45.8258 & 1033 & 3563.85 & 940 & 3243.00 & 1118 & 3857.10 & 837 & 2887.65 \\
\hline 2400 & 48.9898 & 1093 & 3770.85 & 988 & 3408.60 & 1183 & 4081.35 & 876 & 3022.20 \\
\hline 2700 & 51.9615 & 1156 & 3988.20 & 1043 & 3598.35 & 1247 & 4302.15 & 907 & 3129.15 \\
\hline 3000 & 54.7723 & 1202 & 4146.90 & 1080 & 3726.00 & 1325 & 4571.25 & 946 & 3263.70 \\
\hline 3300 & 57.4456 & 1260 & 4347.00 & 1131 & 3901.95 & 1377 & 4750.65 & 978 & 3374.10 \\
\hline 3600 & 60.0000 & 1307 & 4509.15 & 1168 & 4029.60 & 1424 & 4912.80 & 1006 & 3470.70 \\
\hline \multicolumn{2}{|c|}{$\begin{array}{l}\text { Fitting results } \\
\text { between } Z_{i} \text { and } \sqrt{t_{i}}\end{array}$} & \multicolumn{2}{|c|}{$Z_{i}=74.8676+71.23(\mu \mathrm{m})$} & \multicolumn{2}{|c|}{$Z_{i}=60.2687+450.24(\mu \mathrm{m})$} & \multicolumn{2}{|c|}{$Z_{i}=84.8972-95.44(\mu \mathrm{m})$} & \multicolumn{2}{|c|}{$Z_{i}=44.5572+825.43(\mu \mathrm{m})$} \\
\hline \multicolumn{2}{|c|}{$\begin{array}{l}\text { Calculated } D \\
\text { values } \times 10^{-5}\left(\mathrm{~cm}^{2} / \mathrm{s}\right)\end{array}$} & \multicolumn{2}{|c|}{1.1243} & \multicolumn{2}{|c|}{0.7240} & \multicolumn{2}{|c|}{1.4386} & \multicolumn{2}{|c|}{0.6768} \\
\hline \multicolumn{2}{|c|}{$\begin{array}{l}D \text { values in previous } \\
\text { work } \times 10^{-5}\left(\mathrm{~cm}^{2} / \mathrm{s}\right)\end{array}$} & \multicolumn{2}{|c|}{$1.102^{26}$} & \multicolumn{2}{|c|}{$0.737^{27}$} & \multicolumn{2}{|c|}{$1.381^{26}$} & \multicolumn{2}{|c|}{$0.677^{27}$} \\
\hline
\end{tabular}

Table 2. The data of position $Z_{i}$ varied with diffusion time in the four experiments.

layer (the "waist"), which are selected to image clearly on the CCD, are listed in the 2nd row and the 3rd row, respectively. The $4^{\text {th }}$ row shows the relationship between the RI and mass fraction. The $6^{\text {th }}$ to $16^{\text {th }}$ rows are experimental data recorded. The $17^{\text {th }}$ row indicates the fitting results between $Z_{i}$ and $\sqrt{t_{i}}$. The $D$ values can be calculated by comparing the fitting results with Eq. (8), which are listed in the $18^{\text {th }}$ row. The corresponding values in the previous work are listed in the $19^{\text {th }}$ row, which are well consistent with those we reported (the $18^{\text {th }}$ row).

\section{Discussion}

Equation (6) is a fundamental differential equation of diffusion process, derived from the Fick's first law, $F=D \partial C / \partial Z$, and it works only when the $D$ value is a constant. If $D$ depends on the concentration of diffusing substance $C$, the Fick's second law becomes ${ }^{17}$ 


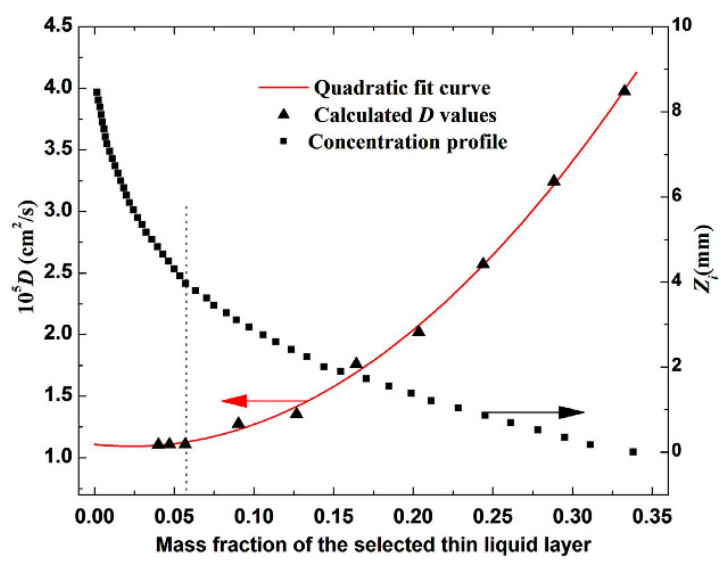

Figure 5. The calculated $D$ values versus the measured concentration profile. Triangle dots: the calculated $D$ values varied with selected thin liquid layers for EG diffusing in water $\left(C_{1}=1, C_{2}=0\right)$. Square dots: concentration profile at the diffusion time of 45 minutes.

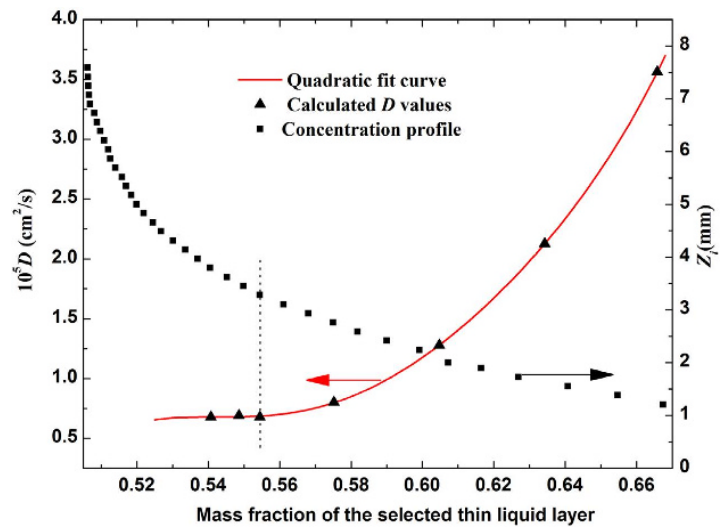

Figure 6. The calculated $D$ values versus the measured concentration profile. Triangle dots: the calculated $D$ values varied with selected thin liquid layers for EG diffusing in $50 \%$ aqueous EG $\left(C_{1}=1, C_{2}=0.5\right)$. Square dots: concentration profile at the diffusion time of 45 minutes.

$$
\frac{\partial C(Z, t)}{\partial t}=\frac{\partial}{\partial Z}\left[D(C) \frac{\partial C(Z, t)}{\partial Z}\right] .
$$

In this case, Eq. (8), the solution of Eq. (6), which is the base of our calculation by observing a thin liquid layer, is untenable. Therefore, the feasibility for calculating $D$ value by Eq.(8) is determined mainly by whether the selected thin liquid layer meets the condition of $D(C) \sim D\left(C_{2}\right)$ in diffusion direction, where $C$ and $C_{2}$ are the concentrations of the thin liquid layer and the destination, respectively. Two experiments have been carried out to verify if the calculated $D$ values are reasonable as follows.

First, the destination concentration is set to be $C_{2}=0$ (infinite dilution), thin liquid layers with different mass fractions have been selected to repeat the experiment (a), the calculated $D$ values are presented by the triangle dots in Fig. 5. The spatial distributions of solution concentration at different diffusion time have been calculated by using Eq. (5) and the resulting diffusion images, the result at diffusion time of $t=45$ minutes is shown in Fig. 5 by the squared dots. It is clear that the calculated $D$ value is indeed dependent on the selected thin liquid layer, provided that when its mass fraction is larger than $C=0.057$, corresponding to the area where concentration profile varies sharply with the diffusion distance, $D(C)>D\left(C_{2}=0\right)$. However, the measured $D$ values tend to be a stable value of $D\left(C_{2}=0\right)=1.1243 \times 10^{-5} \mathrm{~cm}^{2} / \mathrm{s}$ when the selected mass fractions are near the destination $C \rightarrow C_{2}=0$, which corresponds to the area where concentration profile changes smoothly with the diffusion distance, $D(C) \sim D\left(C_{2}=0\right)$. Therefore, the stable value $D\left(C_{2}=0\right)=1.1243 \times 10^{-5} \mathrm{~cm}^{2} / \mathrm{s}$ is reliable in the condition of "infinite dilution".

Second, the destination concentration is set to be $C_{2}=0.50$, liquid layers with different mass fraction have been selected to repeat the experiment (d), and the calculated $D$ values are presented by the triangle dots in Fig. 6 . The spatial distributions of solution concentration at different diffusion time have been calculated by using Eq. (5) and the obtained diffusion images, and the result at diffusion time $t=45$ minutes is shown in Fig. 6 by the squared dots. Figure 6 describes a similar situation as that in Fig. 5, the measured $D$ values in Fig. 6 tend to be a stable 
value of $D=0.6768 \times 10^{-5} \mathrm{~cm}^{2} / \mathrm{s}$ when the mass fractions of selected thin liquid layers are near the destination concentration $C_{2}=0.50$, which is the $D$ value of EG diffusing in the $50 \%$ aqueous EG.

In the method of Holographic interferometry, the concentration difference between two diffusion solutions, $\Delta C=\left(C_{1}-C_{2}\right)$, is required to be small enough ${ }^{19}$ to render $D$ value to be a constant; the Taylor dispersion method only injects a little bit of solute ${ }^{20}$ due to the same reason; there exists a conflict between differential $D$ value measurement accuracy and its initial concentration difference across the diaphragm in classical diaphragm cell technique ${ }^{21}$. While, it is not necessary to maintain a small concentration difference in measuring $D$ value accurately in the method invented by us, because it is fitted well by selecting a suitable thin liquid layer in diffusion cell, which reduces the instrumental requisition in measurement sensitivity. This is a new idea to measure the $D$ values of different solution concentration. On the other hand, the concentration profile can be achieved conveniently based on Eq. (5) benefited from the large initial concentration difference, which appears to offer the potential for applying Boltzmann-Matano ideas ${ }^{22,23}$ to calculate liquid diffusivity of different concentrations, a usual method to measure $D$ value varied with component concentration in solid diffusion, while never used in liquid to the best of our knowledge.

In summary, a new method to measure binary liquid $D$ values based on an ALCL is presented in this paper. Four groups of contrast experiments have been carried out to verify the influence of diffusing substance category, concentration and temperature on diffusion process, and the measured $D$ values are well consistent with data measured by Holographic interferometry and Taylor dispersion methods. The diffusion pool used in this method is more simplified compared with the Taylor dispersion method ${ }^{20}$, the measurement procedure is easier compared with the diaphragm cell technique ${ }^{24}$, and the required experimental environment is more relaxed than that needed in Holographic interferometry method ${ }^{25}$. Further more, this optical method for measuring liquid $D$ value is characterized by visual measurement, short time consuming, and great potential for development, which paves a new way for visual measurements of liquid $D$ values.

\section{References}

1. Jost, W. Diffusion in Solids, Liquids, Gases (Academic 1960).

2. Chhaniwal, V. K. et al. New optical techniques for diffusion studies in transparent liquid solutions. J. Opt. A: Pure Appl. Opt. 5, S329-S337 (2003).

3. Cussler, E. L. Diffusion: Mass Transfer in Fluid Systems, 3rd ed. (Cambridge University, 2009).

4. He, M. G., Zhang, S., Zhang, Y. \& Peng, S. G. Development of measuring diffusion coefficients by digital holographic interferometry in transparent liquid mixtures. Opt. Express 23, 10884-10899 (2015).

5. Byron Bird, R., Stewart, W. E. \& Lightfoot, E. N. Transport Phenomena (John Wiley \& Sons 2002).

6. Riquelme, R., Lira, I., Pérez-López, C., Rayas, J. A. \& Rodríguez-Vera, R. Interferometric measurement of a diffusion coefficient: comparison of two methods and uncertainty analysis. J. Phys. D: Appl. Phys. 40, 2769-2776 (2007).

7. Stoke, R. H. An improved diaphragm-cell for diffusion studies, and some tests of the method. J. Am. Chem. Soc. 72, 763-767 (1950).

8. Leaist, D. G. \& Hao, L. Simultaneous measurement of mutual diffusion and intradiffusion by Taylor dispersion. J. Phys. Chem. 98, 4702-4706 (1994).

9. Zhao, C. W., Lia, J. D. \& Ma, P. S. Diffusion studies in liquids by holographic interferometry. Opt. Laser Technol. 38, 658-662 (2006).

10. Toor, H. L. Diffusion measurements with a diaphragm cell. J. Phys. Chem. 64, 1580-1582 (1960).

11. Cipelletti, L., Biron, J. P., Martin, M. \& Cottet, H. Measuring arbitrary diffusion coefficient distributions of Nano-Objects by Taylor dispersion analysis. Anal. Chem. 87, 8489-8496 (2015).

12. Guo, Z. X., Maruyama, S. \& Komiya, A. Rapid yet accurate measurement of mass diffusion coefficients by phase shifting interferometer. J. Phys. D: Appl. Phys. 32, 995-999 (1999).

13. Weingärtner, H. Diffusion in liquid mixtures of light and heavy water. Ber. Bunsenges. Phys. Chem. 88, 47-50 (1984).

14. Ven-Lucassen, I. M. J. J., Kieviet, F. G. \& Kerkhof, P. J. A. M. Fast and convenient implementation of the Taylor dispersion method. J. Chem. Eng. Data 40, 407-411 (1995).

15. Sun, L. C. \& Pu, X. Y. Asymmetric liquid-core cylindrical lens used to measure liquid diffusion coefficient. Appl. Opt. 55, 2011-2017 (2016).

16. Sun, L. C., Meng, W. D. \& Pu, X. Y. New method to measure liquid diffusivity by analyzing an instantaneous diffusion image. Opt. Express 23, 23155-23166 (2015).

17. Crank, J. The Mathematics of Diffusion (Oxford University 1975).

18. Abramowitz, M. \& Stegun, I. A. Handbook of Mathematical Functions with Formulas, Graphs and Mathematical Tables (Dover 1972).

19. Ruiz-Bevia, F., Celdran-Mallol, A., Santos-Garcia, C. \& Fernandez-Sempere, J. Holographic interferometric study of free diffusion: a new mathematical treatment. Appl. Opt. 24, 1481-1484 (1985).

20. Funazukuri, T., Sugihara, T., Yui, K., Ishii, T. \& Taguchi, M. Measurement of infinite dilution diffusion coefficients of vitamin $\mathrm{K}_{3}$ in $\mathrm{CO}_{2}$ expanded methanol. J. of Supercritical Fluids 108, 19-25 (2016).

21. Hansjürgen Schönert, J. K. Diffusion in the Diaphragm Cell: Continuous monitoring of the concentrations and determination of the differential diffusion coefficient. J. Solution Chem. 43, 71-82 (2014).

22. Palcut, M., Knibbe, R., Wiik, K. \& Grande, T. Cation inter-diffusion between $\mathrm{LaMnO}_{3}$ and $\mathrm{LaCoO}_{3}$ materials. Solid State Ion. 202, 6-13 (2011).

23. Schnabel, M., Siddique, A. B., Janz, S. \& Wilshaw, P. R. Phosphorus diffusion in nanocrystalline 3C-SiC. Appl. Phys. Lett. 106, 133101 (2015).

24. Antwi, M. K., Myerson, A. S. \& Zurawsky, W. Diffusion of lysozyme in buffered salt solutions. Ind. Eng. Chem. Res. 50, 10313-10319 (2011).

25. Chhaniwal, V. K., Anand, A. \& Narayanamurthy, C. S. Measurement of diffusion coefficient of transparent liquid solutions using Michelson interferometer. Opt. Lasers Eng. 42, 9-20 (2004).

26. Wang, M. H., Soriano, A. N., Caparanga, A. R. \& Li, M. H. Mutual diffusion coefficients of aqueous solutions of some glycols. Fluid Phase Equilib. 285, 44-49 (2009).

27. Fernández-Sempere, J., Ruiz-Beviá, F., Colom-Valiente, J. \& Más-Pérez, F. Determination of diffusion coefficients of glycols. J. Chem. Eng. Data 41, 47-48 (1996).

\section{Acknowledgements}

We acknowledge the support from the National Science Foundation of China (Grant Nos 11164033 and 61465014). 


\section{Author Contributions}

L.S. designed and carried out the experiments and wrote the manuscript. X.P. conceived the project and revised the manuscript.

\section{Additional Information}

Supplementary information accompanies this paper at http://www.nature.com/srep

Competing financial interests: The authors declare no competing financial interests.

How to cite this article: $\mathrm{Sun}, \mathrm{L}$. and $\mathrm{Pu}, \mathrm{X}$. A novel visualization technique for measuring liquid diffusion coefficient based on asymmetric liquid-core cylindrical lens. Sci. Rep. 6, 28264; doi: 10.1038/srep28264 (2016).

(c) (i) This work is licensed under a Creative Commons Attribution 4.0 International License. The images or other third party material in this article are included in the article's Creative Commons license, unless indicated otherwise in the credit line; if the material is not included under the Creative Commons license, users will need to obtain permission from the license holder to reproduce the material. To view a copy of this license, visit http://creativecommons.org/licenses/by/4.0/ 